\title{
3D continuum radiative transfer in complex dust configurations around stellar objects and active galactic nuclei
}

\section{Computational methods and capabilities}

\author{
J. Steinacker ${ }^{1}$, Th. Henning ${ }^{2}$, A. Bacmann ${ }^{3}$, and D. Semenov ${ }^{1}$ \\ 1 Astrophysical Institute and University Observatory (AIU), University of Jena, Schillergässchen 2-3, 07745 Jena, Germany \\ e-mail: stein@astro.uni-jena.de;dima@astro.uni-jena.de \\ 2 Max Planck Institute for Astronomy, Königstuhl 17, 69117 Heidelberg, Germany \\ e-mail: henning@mpia-hd.mpg.de \\ 3 European Southern Observatory, Karl-Schwarzschild-Str. 2, 85748 Garching, Germany \\ e-mail: abacmann@eso.org
}

Received 23 September 2002 / Accepted 9 December 2002

\begin{abstract}
We present the new grid-based code SteInRaY which has been developed to solve the full 3D continuum radiative transfer problem generally arising in the analysis of star-forming regions, matter around evolved stars, starburst galaxies, or tori around active galactic nuclei. The program calculates the intensity emerging from these complicated structures using a combination of step-size controlled ray-tracing and adaptive multi-wavelength photon transport grids. Along with a 2nd order finite differencing approach, the grids are optimized to reduce the discretization error and provide global error control. The full wavelength-dependent problem is solved without any flux approximation, and for arbitrary scattering properties of the dust. The program is designed to provide spatially resolved images, visibilities, and spectra of complex dust distributions even without any symmetry for wavelengths ranging from the UV to FIR and allows for multiple internal and external sources. In this paper, the algorithm is described and the capabilities of the code are illustrated by the treatment of 1D and 3D test cases. Analyzing the properties of typical cosmic dust, we discuss the wavelength range for which the time-consuming solution on adaptive grids can be omitted. The temperature is calculated self-consistently using standard accelerated $\Lambda$-iteration.
\end{abstract}

Key words. radiative transfer - methods: numerical - accretion, accretion disks - ISM: dust extinction

\section{Introduction}

Nearly all information about astrophysical sources comes from analyzing the radiation we receive from the objects. Therefore, radiative transport (thereafter RT) is one of the most fundamental processes considered in astrophysics whenever radiation is altered on its way from the source to the telescope. The alteration can range from small perturbations like the reddening of stellar light due to interstellar extinction or the change of polarization due to the Faraday effect, to almost complete shielding at short wavelengths and thermal re-emission at infrared and sub-millimeter wavelengths in the case of deeplyembedded star-forming regions, envelopes, and evolved stars, or tori around active galactic nuclei.

In view of the complex structure of the matter around these objects revealing filamentary, disk- or ring-like distributions, simple 1D approximations often fail to describe the observed images and spectral energy distributions, making

Send offprint requests to: J. Steinacker, e-mail: stein@astro.uni-jena.de multi-dimensional RT mandatory. But even for symmetric dust distributions, asymmetric source distributions or boundary conditions like, e.g., a single star near the dust cloud boundary have to be treated with 3D RT. This will be even more necessary in the near future, as the oncoming interferometers such as VLTI, Keck, and ALMA will reach resolutions revealing details at milli-arcsecond level, hence resolving complicated 3D structures like planetary gaps (Bryden et al. 1999; D'Angelo et al. 2002), density waves (Pfalzner et al. 2000), interaction streamers (Günther \& Kley 2002), and disk warps (Bouvier et al. 1999).

But it is not only for explaining complex observational data that flexible three-dimensional RT programs are required. 3D Smooth-Particle Hydrodynamical (SPH) and MagnetoHydrodynamical (MHD) simulations are now available and produce time-dependent density and temperature distributions of dust and gas in star-forming regions, proto-planetary disks, and in dust tori around AGNs. Without a 3D RT code, it is impossible to predict at which wavelengths or by which telescope these 3D features can be detected. Moreover, only with a 3D 
RT program can the validity of the approximate RT used in 3D SPH/MHD simulations be verified.

In line RT, the existing codes often use coarse approximations for the spatial resolution and the scattering to be able to handle the numerically intensive calculation of the level population (e.g. Folini \& Walder 1999; Uitenbroek 2001; and references therein).

For 3D continuum RT, different methods have been applied to solve the complex transport equation, but only a few codes are available. The advantage of Monte-Carlo methods is the easy treatment of complex density distributions, complicated scattering functions, and polarization. Monte-Carlo methods require high numbers of photons to cover re-emission in all directions and to treat optically thick regions, which can be reduced using elaborated concepts like immediate reradiation. The major drawback, however, is that there is no global error control when using Monte-Carlo schemes. They have been applied for 3D configurations in the papers of Egan \& Shipman (1995), Wolf et al. (1999), Wolf \& Henning (1999) incorporating polarization, and Gordon et al. (2001), Misselt et al. (2001) considering also transient heating.

Integrating the RT equation along the considered line of sight by using a ray-tracer is a very flexible method for treating complex density configurations. It provides full error control, but requires the implicit re-calculation of the step size and becomes very time-consuming when the optical depth is high, so that approximations have to be used. The first solution was published by Yorke (1986) solving the wavelengthdependent problem in flux-limited approximation and without self-consistent temperature iteration.

Well-established in hydrodynamical simulations, solutions on fixed grids can also be performed in RT using a finite difference or short characteristics discretization. However, resolution of all relevant structures for all wavelengths is very hard to achieve for most astrophysical applications. Given that the intensity depends on wavelength, three spatial and two directional coordinates, a decent resolution of 100 points in each variable already leads to solution vectors with $10^{12}$ components, which is not possible to store simultaneously even in supercomputers nowadays. Together with the complicated integro-differential type of the RT equation which makes application of common sparse matrix solvers impossible, a solution of the 3D RT can only be achieved by using sophisticated adaptive grids and fast solution algorithms. Two 3D continuum RT grid codes are available. Stenholm et al. (1991) solved the transport equation on a regular grid using 1st order finite differencing with low spatial resolution. Folini et al. (2003) have developed a code to solve the optically thick, stationary, wavelength decoupled, and unpolarized NLTE radiative transfer problem for moving media of a given density.

This publication is the second in a series presenting the new grid-based 3D RT code STEINRAY which incorporates the use of multi-wavelength adaptive photon transport grids to provide global error control of the solution. The underlying grid generation mechanism was described in Steinacker et al. (2002b). In this paper, the full discretization and solution strategy is given including the self-consistent temperature iteration, as well as simple test cases to show the capabilities of the code. In another paper, we compare the results for a standard accretion disk in a 2D benchmark project with three other RT codes (Pascucci et al. 2003). Further publications will deal with applications to circumbinary disks, warped disks, gaps in disks, pre-stellar cores, and dust tori around AGNs.

In Sect. 2, the continuum RT equation is described and the solution strategy is outlined. The optical properties of typical cosmic dust are briefly reviewed in Sect. 3 and limiting wavelengths are derived to decide when time-consuming calculations on the adaptive multi-wavelengths photon transport grids have to be performed. The solution of the transport equation in the case of substantial scattering is described in Sect. 4. Section 5 describes the discretization of the temperature distribution. The code is tested in Sect. 6 for simple 1D cases and a complex 3D application. We summarize our findings in Sect. 7.

\section{The 3D continuum transport equation}

We describe the radiation field by the total specific intensity $\mathcal{I}_{\lambda}(\lambda, \boldsymbol{x}, \boldsymbol{n})$, where $\boldsymbol{x}$ gives the location in space, $\boldsymbol{n}$ is the direction of the radiation, and $\lambda$ its wavelength. Starting with the boundary values, we can calculate the transport of the radiation through the considered domain by solving the stationary 3D RT equation

$$
\begin{aligned}
\boldsymbol{n} \nabla_{\boldsymbol{x}} \mathcal{I}(\lambda, \boldsymbol{x}, \boldsymbol{n})= & -\left[\kappa^{\mathrm{abs}}(\lambda, \boldsymbol{x})+\kappa^{\mathrm{sca}}(\lambda, \boldsymbol{x})\right] \mathcal{I}(\lambda, \boldsymbol{x}, \boldsymbol{n}) \\
& +\kappa^{\mathrm{abs}}(\lambda, \boldsymbol{x}) B[\lambda, T(\boldsymbol{x})] \\
& +\frac{\kappa^{\mathrm{sca}}(\lambda, \boldsymbol{x})}{4 \pi} \int_{\Omega} \mathrm{d} \Omega^{\prime} p\left(\lambda, \boldsymbol{n}, \boldsymbol{n}^{\prime}\right) \mathcal{I}\left(\lambda, \boldsymbol{x}, \boldsymbol{n}^{\prime}\right)
\end{aligned}
$$

with the Planck function $B$, the dust temperature $T$, and the phase function $p$. For simplicity, we will skip the $\lambda$ index of the intensity which indicates that it is defined per wavelength interval. The extincting properties of enshrouding matter are described by the line and continuum absorption and scattering of the radiation by dust grains and gas species

$\kappa^{\mathrm{abs}, \mathrm{sca}}(\lambda, \boldsymbol{x})=\kappa_{\mathrm{gas}}^{\mathrm{abs}, \mathrm{sca}}(\lambda, \boldsymbol{x})+\kappa_{\mathrm{dust}}^{\mathrm{abs}, \mathrm{sca}}(\lambda, \boldsymbol{x})$.

In this paper, we focus on the solution of (1) for continuum radiation, and consider the absorption and scattering by dust only, omitting the subscript "dust" in the rest of the text for clarity. We will restrict our consideration to the use of dust particles with one size and a specific chemical composition. Equation (1) can also be used for a size or composition distribution of dust grains, but then each of the different dust species will have its own temperature.

The physical quantities describing the efficiency to absorb and scatter the incident radiation by an ensemble of dust grains can be written as

$\kappa^{\mathrm{abs}, \mathrm{sca}}(\lambda, \boldsymbol{x})=k^{\mathrm{abs}, \mathrm{sca}}(\lambda, \boldsymbol{x}) \rho(\boldsymbol{x})$,

where $\rho(\boldsymbol{x})$ is the gas density and $k^{\text {abs,sca }}(\lambda, \boldsymbol{x})$ are the mass absorption and scattering coefficients of the dust ensemble, respectively.

Two source terms of radiation are explicitely given in Eq. (1), namely scattering into the line of sight and re-radiation 
by the dust particles. The scattering into the direction $\boldsymbol{n}$ is described by the probability $p\left(\lambda, \boldsymbol{n}, \boldsymbol{n}^{\prime}\right)$ that radiation is scattered from the direction $\boldsymbol{n}^{\prime}$ into $\boldsymbol{n}$, with the solid angle $\Omega^{\prime}$. The second source is the re-radiation of the dust particles $\kappa^{\mathrm{abs}} B$.

Intensity and dust temperature are not independent. The radiation field determines the temperature and in turn, the dust re-emission contributes to the radiation field. This couples the partial integro-differential RT equation to the local energy balance equation describing how a dust particle is heated by the source radiation and the radiation of all other particles. The balance equation for the energy density in local thermal equilibrium at point $\boldsymbol{x}$ is

$$
\begin{aligned}
\int_{0}^{\infty} \mathrm{d} \lambda Q^{\mathrm{abs}}(\lambda) B\left[\lambda, T_{\mathrm{rad}}(\boldsymbol{x})\right]= & \int_{0}^{\infty} \mathrm{d} \lambda Q^{\mathrm{abs}}(\lambda) \frac{1}{4 \pi} \\
& \times \int_{\Omega} \mathrm{d} \Omega^{\prime} \mathcal{I}\left(\lambda, \boldsymbol{x}, \boldsymbol{n}^{\prime}\right) .
\end{aligned}
$$

The temperature is denoted by $T_{\text {rad }}$ to distinguish it from temperatures arising from other possible heating sources like viscous heating, cosmic rays, or gas-grain collisions.

A simultaneous treatment of the 3D RT and hydrodynamical (HD) equations in one code is currently beyond the capabilities of nowadays computers. HD simulations commonly use an approximate RT to calculate radiative heating, while in turn, RT codes can use the derived densities and the heating sources to calculate the radiation field at a given time or in a stationary picture.

In view of the substantial computational effort to solve the 3D transport equation, it is mandatory to use any approximation that is allowed by the physical conditions. With vanishing scattering integral, (1) becomes a 1st order differential equation which can be solved without problems using ray-tracing. To make use of this approximation, and since the operators in the integro-differential Eq. (1) are linear in $\mathcal{I}$, we can (following e.g. Efstathiou \& Rowan-Robinson 1990) split the total specific intensity into an unprocessed passing source component $I^{*}$ that includes also the thermal contribution from the dust, and a processed component $I$ of radiation that has encountered scattering

$$
I=I^{*}+I \text {. }
$$

Substituting the source term to be

$$
\begin{aligned}
S(\lambda, \boldsymbol{x}, \boldsymbol{n}) \equiv & \frac{1}{4 \pi} \kappa^{\mathrm{sca}}(\lambda, \boldsymbol{x}) \int_{\Omega} \mathrm{d} \Omega^{\prime} p\left(\lambda, \boldsymbol{n}, \boldsymbol{n}^{\prime}\right) I\left(\lambda, \boldsymbol{x}, \boldsymbol{n}^{\prime}\right) \\
& +C^{*}(\lambda, \boldsymbol{x}, \boldsymbol{n}),
\end{aligned}
$$

this leads to three equations

$$
\begin{aligned}
& \boldsymbol{n} \nabla_{\boldsymbol{x}} I^{*}(\lambda, \boldsymbol{x}, \boldsymbol{n})=-\kappa^{\mathrm{ext}}(\lambda, \boldsymbol{x}) I^{*}(\lambda, \boldsymbol{x}, \boldsymbol{n})+\kappa^{\mathrm{abs}}(\lambda, \boldsymbol{x}) B[\lambda, T(\boldsymbol{x})] \\
& \boldsymbol{n} \nabla_{\boldsymbol{x}} I(\lambda, \boldsymbol{x}, \boldsymbol{n})=-\kappa^{\mathrm{ext}}(\lambda, \boldsymbol{x}) I(\lambda, \boldsymbol{x}, \boldsymbol{n})+S(\lambda, \boldsymbol{x}, \boldsymbol{n}) \\
& \int_{0}^{\infty} \mathrm{d} \lambda Q^{\mathrm{abs}}(\lambda) B[\lambda, T(\boldsymbol{x})]=\int_{0}^{\infty} \mathrm{d} \lambda Q^{\mathrm{abs}}(\lambda) \frac{1}{4 \pi} \\
& \quad \times \int_{\Omega} \mathrm{d} \Omega^{\prime} I\left(\lambda, \boldsymbol{x}, \boldsymbol{n}^{\prime}\right)+D^{*}(\boldsymbol{x})
\end{aligned}
$$

with the known contributions

$$
\begin{aligned}
& C^{*}(\lambda, \boldsymbol{x}, \boldsymbol{n})=\frac{\kappa^{\mathrm{sca}}(\lambda, \boldsymbol{x})}{4 \pi} \int_{\Omega} \mathrm{d} \Omega^{\prime} p\left(\lambda, \boldsymbol{n}, \boldsymbol{n}^{\prime}\right) I^{*}\left(\lambda, \boldsymbol{x}, \boldsymbol{n}^{\prime}\right) \\
& D^{*}(\boldsymbol{x})=\int_{0}^{\infty} \mathrm{d} \lambda Q^{\mathrm{abs}}(\lambda) \frac{1}{4 \pi} \int_{\Omega} \mathrm{d} \Omega^{\prime} I^{*}\left(\lambda, \boldsymbol{x}, \boldsymbol{n}^{\prime}\right)
\end{aligned}
$$

and the abbreviation $\kappa^{\mathrm{ext}}=\kappa^{\mathrm{abs}}+\kappa^{\mathrm{sca}} \cdot C^{*}(\lambda, \boldsymbol{x}, \boldsymbol{n})$ represents the source and thermal radiation that is scattered at the point $\boldsymbol{x}$ into the direction $\boldsymbol{n}$. The source and thermal contribution to the heating is described by $D^{*}(\boldsymbol{x})$.

As intended by the split (5), the first transfer Eq. (7) can be transformed to a path integral and thus easily be calculated using the formal exponential solution. For an empirical optical data set, the numerical solution is conveniently derived for all wavelengths e.g. using fifth-order Runge-Kutta with adaptive stepsize control (Press et al. 1992) as ray-tracing routine. This provides error control for the solution, and can be used for all optical depths from thin to thick regions. Moreover, it allows to treat multiple external and internal sources of radiation.

The second Eq. (8) still has an integro-differential form and requires a separate, more sophisticated treatment. The third Eq. (9) allows to update the temperature from an intensity that has been calculated assuming a fixed temperature using a standard accelerated $\Lambda$-iteration ( $\mathrm{Ng}$ 1974).

\section{Limiting wavelength for the consideration of scattering}

In order to decide where the scattering integral becomes negligible and when the solution of the RT equation $I^{*}$ can be calculated by solving (7) easily, the optical properties of the dust grains have to be investigated. For the sake of comparison, we concentrate on homogeneous spherical particles of a uniform size (radius $a=0.1 \mu \mathrm{m}$ ) consisting of amorphous carbon (Preibisch et al. 1993; Rouleau \& Martin 1991) or astro-silicate (Draine \& Lee 1984; Draine 1985). Their optical properties are calculated by the Mie theory for spheres (Mie 1908) using the modified code of Barber \& Hill (1990).

More complicated dust models like mixtures of (in)homogeneous spherical particles of various sizes and compositions can be treated by the code without difficulty, but at expense of longer computational time.

To illustrate the typical behavior of the optical properties, we show in Fig. 1 the ratio of the scattering and absorption efficiency factors of dust particles consisting of amorphous carbon for different sizes $a$ as a function of wavelength. The ratio roughly remains around 1 for wavelengths smaller than the particle size and drops for larger wavelengths following $\mathrm{a} \sim \lambda^{-2.8}$. powerlaw. In order to give a conservative criterion for neglecting scattering, we define the limiting wavelength $\lambda_{\mathrm{s}}$ to be the wavelength where the ratio has dropped to $10^{-2}$. For larger wavelengths, we can omit solving (8) and directly derive the total intensity from (7). In Fig. 2, the limiting wavelength is plotted against particle size $a$ for amorphous carbon (dotted line) and astro-silicate (solid line). Roughly following an $a^{1.12}$. powerlaw, the line indicates that for optical wavelengths, all 


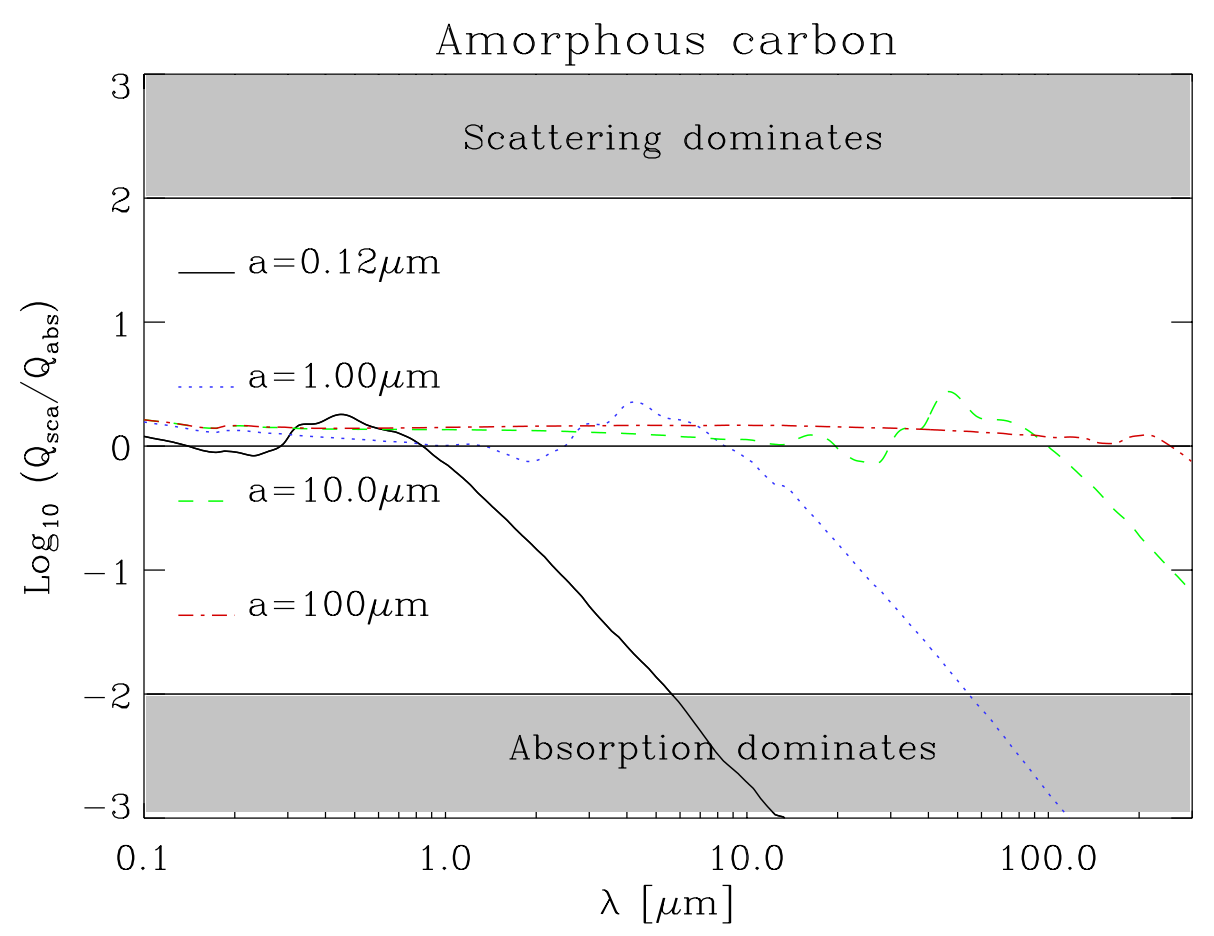

Fig. 1. Ratio of scattering and absorption efficency factor as function of the wavelength. The different curves correspond to different particle sizes $a$. Two solid lines at the values 2 and -2 limit the regions where scattering and absorption is considered to dominate, respectively.

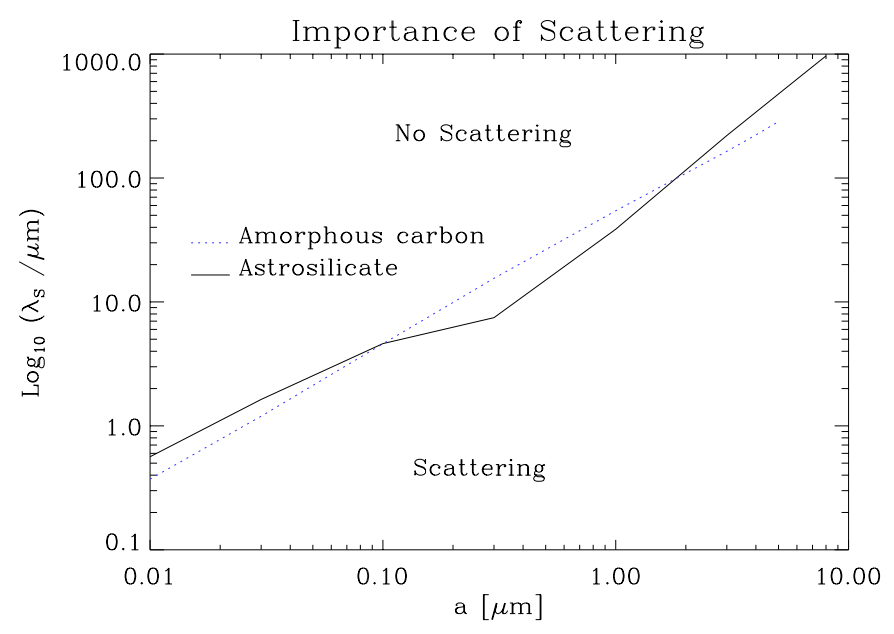

Fig. 2. Limiting wavelength for which scattering can be neglected, plotted as a function of particle size $a$ for amorphous carbon (dotted) and astro-silicate (solid). In the parts above the lines, absorption is more than a factor of 100 stronger than scattering.

particles of astrophysical relevance larger than $0.01 \mu \mathrm{m}$ scatter the radiation. In the near IR, particles with $a>0.1 \mu \mathrm{m}$ require scattering calculations. But even at higher wavelengths around $100 \mu \mathrm{m}$, particles larger than $2 \mu \mathrm{m}$ will scatter the radiation substantially.

Depending on a given particle size $a$ and wavelength $\lambda$, the program switches from direct solution of the scattering Eqs. (7)-(9) for $\lambda<\lambda_{\mathrm{s}}$ to the easier ray-tracing solution of (7) for $\lambda>\lambda_{\mathrm{s}}$ with the limiting wavelength $\lambda_{\mathrm{s}}=100 a^{1.12}$.

If scattering is important, beside the scattering cross section, the phase function $p\left(\lambda, \boldsymbol{n}, \boldsymbol{n}^{\prime}\right)$ describing the probability of the incoming radiation to be scattered from the direction $\boldsymbol{n}^{\prime}$ into $\boldsymbol{n}$ also has to be specified. This is important, as for isotropic scattering, when $p\left(\lambda, \boldsymbol{n}, \boldsymbol{n}^{\prime}\right)=1$, (1) can be simplified. Integrating over the solid angle, the scattering integral disappears and the equation becomes an ordinary differential equation

$\boldsymbol{n} \nabla_{\boldsymbol{x}} \mathcal{J}(\lambda, \boldsymbol{x})=\kappa^{\mathrm{abs}}(\lambda, \boldsymbol{x})[B(\lambda, \boldsymbol{x})-\mathcal{J}(\lambda, \boldsymbol{x})]$

for the total mean intensity

$\mathcal{J}(\lambda, \boldsymbol{x})=\frac{1}{4 \pi} \int_{\Omega} \mathrm{d} \Omega^{\prime} \mathcal{I}\left(\lambda, \boldsymbol{x}, \boldsymbol{n}^{\prime}\right)$

which is easy to solve. We show in Fig. 3 the phase function in polar coordinates for homogenous silicate spheres of size $a=1 \mu \mathrm{m}$ for different wavelengths. Radiation that is traveling parallel to the $x$-axis will be scattered by the dust particle at the origin with a probability $p$ into a direction described by $\theta$. Commonly, also the size parameter $x \equiv 2 \pi a / \lambda$ is used which we give in the picture, as well as the complex refractive index $m_{\lambda}$ of the silicate. Evidently, in the optical wavelength range, forward scattering dominates, making the direct use of any approximation like isotropic scattering impossible. But even for the limiting case of Rayleigh scattering $\left(\frac{2 \pi a}{\lambda}\left|m_{\lambda}\right| \ll 1\right)$, for which the phase function is most symmetric, the ratio of the probability of the radiation to be scattered in forward and in perpendicular direction still equals 2, as can be seen in Fig. 4 (dash-dotted line). Here, we have also plotted the phase function for a smaller particle $a=0.12 \mu \mathrm{m}$ for different wavelengths.

Anisotropic scattering can become very important. If the dust grains are, e.g., of size $1 \mu \mathrm{m}$, in the optically thin case, 


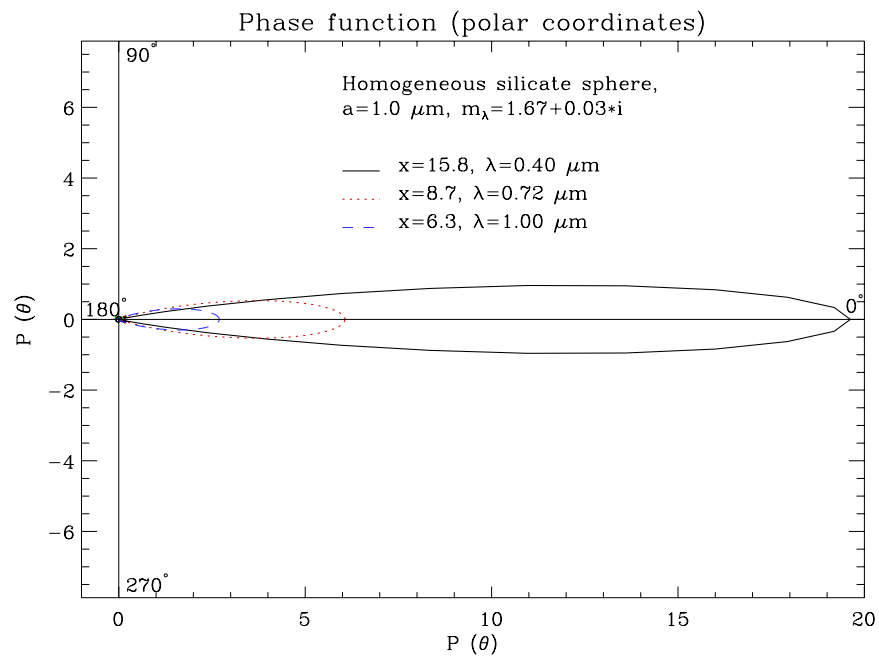

Fig. 3. Scattering phase function in polar coordinates for homogenous spherical silicate particle of size $a=1 \mu \mathrm{m}$ for different wavelengths. $m_{\lambda}$ denotes the mean complex refractive index for all three plotted wavelengths and $x=2 \pi a / \lambda$ is the size parameter. In the optical wavelength range, the scattering is strongly peaked in the forward direction.

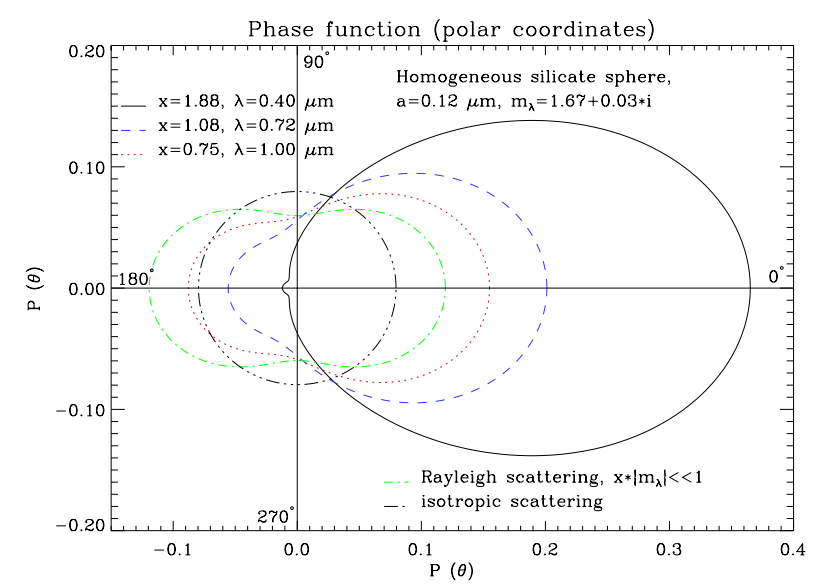

Fig. 4. Scattering phase function in polar coordinates for homogenous spherical silicate particle of size $a=0.12 \mu \mathrm{m}$ for different wavelengths. $m_{\lambda}$ denotes the mean complex refractive index for all three plotted wavelengths and $x=2 \pi a / \lambda$ is the size parameter. In the optical wavelength range, the scattering is peaked in the forward direction, but approaches the Rayleigh scattering limit where the ratio between forward and perpendicular scattered intensities becomes 2 , for longer wavelengths.

there will be just a few scattering events before the radiation escapes the object, and the scattered radiation is strongly peaked in the forward direction. Assuming isotropic scattering, the scattered radiation will be more homogeneous and substantially different from the anisotropic case. We just mention as one prominent example the atmosphere of an accretion disk where the matter has low density and just a few scattering events occur before the radiation escapes the object. In particular, interpreting images obtained for edge-on accretion disks at short wavelengths is impossible without using the correct anisotropic phase function. In the program, we always use the correct anisotropic phase function.
If more than one dust size is considered, the phase function may differ substantially between the different sizes. For efficient mixing of the dust grains (assuming the same density distribution for all sizes), a mean scattering function may be defined to handle the transport of the scattered part of the radiation approximatively.

\section{Discretization and solution strategy of the RT equation with scattering}

In the following, we describe how the integro-differential Eq. (8) is discretized and solved in the program. For the direction coordinates $\boldsymbol{n}$, we choose spherical coordinates $\vartheta, \varphi$, while Cartesian coordinates $x, y, z$ are best suited for spatial coordinates in 3D RT problems with complex density distributions. Hence, (8) turns into

$$
\begin{aligned}
& \sin \vartheta \cos \varphi \frac{\partial I(\lambda, x, y, z, \vartheta, \varphi)}{\partial x}+\sin \vartheta \sin \varphi \frac{\partial I(\lambda, x, y, z, \vartheta, \varphi)}{\partial y} \\
& \quad+\cos \vartheta \frac{\partial I(\lambda, x, y, z, \vartheta, \varphi)}{\partial z}=-\kappa^{\mathrm{ext}}(\lambda, x, y, z) I(\lambda, x, y, z, \vartheta, \varphi) \\
& \quad+S(\lambda, x, y, z, \vartheta, \varphi)
\end{aligned}
$$

It is likely that close to the source, the radiation field will be peaked in a certain direction. To minimize discretization errors, the direction grid should be refined around this $\boldsymbol{n}$. But as this refinement strongly varies with the location, a coupled location and direction grid would have to be used, which is numerically prohibitive. Hence, we choose a direction grid that is equally spaced on the unit sphere with as many grid points as can be afforded numerically. Steinacker et al. (1996) have calculated equally spaced nodes for the cubature of the unit sphere and corresponding weights derived by evaluating special Gegenbauer polynomials. The grid points have been obtained using a special Metropolis algorithm to maximize the distance between the nodes. We denote the number of nodes with $N_{\vartheta \varphi}$, and typical numbers we use range between 49 and 400 for a dust particle size of $0.12 \mu \mathrm{m}$. In view of the strongly peaked phase function for larger dust particles, higher numbers might be necessary to resolve the peak appropriately. For the wavelength, we introduce a logarithmic grid $\lambda^{f}=\lambda^{0} \times(\Delta \lambda)^{f}$ (in $\lambda^{f}, f$ is an index, while $(\Delta \lambda)^{f}$ denotes $\Delta \lambda$ to the power of $f$ ) with $N_{\lambda}$ grid points, and use trapezoidal logarithmic integration in all occurring integrals.

For the spatial discretization, we use the adaptive optimized multi-wavelength photon transport grids presented in Steinacker et al. (2002b). These grids are obtained with a refinement grid generator to minimize the 1st order discretization error and guarantee global error control for the solution of the radiative transfer problem on the grid. For each wavelength grid point, a spatial grid is calculated, so that the number $N_{\mathrm{sp}}^{f}$ of grid points per grid depends on the wavelength. The three Cartesian coordinates are $x_{s}^{f}, y_{s}^{f}, z_{s}^{f}$, respectively, used at wavelength grid point $f$ where $s=1, \ldots, N_{\mathrm{sp}}^{f}$. The numbering of the grid points by $s$ is caused by the refinement procedure which generates the adaptive grids. They will be renumbered with respect to the boundary conditions and the considered direction. 
The intensity is therefore discretized by a solution vector with $N_{\vartheta \varphi} \sum_{f=1, N_{\lambda}} N_{\mathrm{sp}}^{f}$ components, reaching values around $10^{6}$ for typical astrophysical applications. Each vector component of the intensity can be written as

$I_{s}^{f, g} \equiv I\left(\lambda^{f}, x_{s}^{f}, y_{s}^{f}, z_{s}^{f}, \vartheta^{g}, \varphi^{g}\right)$.

The derivative $\boldsymbol{n} \nabla_{\boldsymbol{x}} I(\lambda, \boldsymbol{x}, \boldsymbol{n})$ in (8) has to be calculated on the spatial grid in order to treat the transport of scattered radiation properly. Steinacker et al. (2002a) have shown that 1st order finite differencing schemes introduce numerical diffusion into the solution of the RT equation, completely blurring the features of the solution. We choose 2 nd order finite differencing to approximate the derivative, as 3 rd order finite differencing schemes have been shown in that paper to be too timeconsuming for 3D RT.

The notation for a use of a 2 nd order finite differencing scheme on an adaptively refined grid is shown in Fig. 5a. To calculate the unknown intensity at a given point $\boldsymbol{A}=\left(x_{s}^{f}, y_{s}^{f}, z_{s}^{f}\right)$ in the grid, we use the known intensities at the two preceding grid points along the Cartesian axes, with respect to the considered ray propagation direction (we indicated this direction in the small coordinate system in the lower right corner of Fig. 5b). In this notation, e.g. the point preceding $A$ along the $x$-axis has the $x$-coordinate $x_{-1}$. The set of seven points defines a stencil that is moved through the grid in the course of solving the transport equation for the scattered radiation. As shown in Fig. 5a, this may lead to situations where for a point $\boldsymbol{A}$ there is no second preceding grid point. In that case, 8-point interpolations are performed for the preceding cube to obtain the intensity at $C$.

In 2nd order approximation, the derivatives with respect to the point $\left(x_{s}^{f}, y_{s}^{f}, z_{s}^{f}\right)$ and suppressing the $s$ index read as

$$
\begin{aligned}
& \left.\frac{\partial I}{\partial x}\right|_{x^{f}} \approx \\
& \frac{I_{0,0,0}^{f, g} \Delta x_{-2}^{f}\left[2 \Delta x_{-1}^{f}+\Delta x_{-2}^{f}\right]-I_{-1,0,0}^{f, g}\left[\Delta x_{-2}^{f}+\Delta x_{-1}^{f}\right]^{2}+I_{-2,0,0}^{f, g}\left(\Delta x_{-1}^{f}\right)^{2}}{\left(x^{f}\right)^{2} \Delta x_{-2}^{f}-\left(x_{-1}^{f}\right)^{2}\left[\Delta x_{-2}^{f}+\Delta x_{-1}^{f}\right]+\left(x_{-2}^{f}\right)^{2} \Delta x_{-1}^{f}}
\end{aligned}
$$

$\left.\frac{\partial I}{\partial y}\right|_{y^{f}} \approx$

$$
\frac{I_{0,0,0}^{f, g} \Delta y_{-2}^{f}\left[2 \Delta y_{-1}^{f}+\Delta y_{-2}^{f}\right]-I_{0,-1,0}^{f, g}\left[\Delta y_{-2}^{f}+\Delta y_{-1}^{f}\right]^{2}+I_{0,-2,0}^{f, g}\left(\Delta y_{-1}^{f}\right)^{2}}{\left(y^{f}\right)^{2} \Delta y_{-2}^{f}-\left(y_{-1}^{f}\right)^{2}\left[\Delta y_{-2}^{f}+\Delta y_{-1}^{f}\right]+\left(y_{-2}^{f}\right)^{2} \Delta y_{-1}^{f}}
$$

$$
\begin{aligned}
& \left.\frac{\partial I}{\partial z}\right|_{z^{f}} \approx \\
& \frac{I_{0,0,0}^{f, g} \Delta z_{-2}^{f}\left[2 \Delta z_{-1}^{f}+\Delta z_{-2}^{f}\right]-I_{0,0,-1}^{f, g}\left[\Delta z_{-2}^{f}+\Delta z_{-1}^{f}\right]^{2}+I_{0,0,-2}^{f, g}\left(\Delta z_{-1}^{f}\right)^{2}}{\left(z^{f}\right)^{2} \Delta z_{-2}^{f}-\left(z_{-1}^{f}\right)^{2}\left[\Delta z_{-2}^{f}+\Delta z_{-1}^{f}\right]+\left(z_{-2}^{f}\right)^{2} \Delta z_{-1}^{f}},
\end{aligned}
$$

with $I_{j, k, l}^{f, g}=I^{f, g}\left(x_{j}^{f}, y_{k}^{f}, z_{l}^{f}\right)$. To abbreviate, we define the constants

$$
A_{r}^{f} \equiv\left(\Delta r_{-2}^{f}\right)^{2}
$$

$$
\begin{aligned}
B_{r}^{f} & \equiv\left(\Delta r_{-1}^{f}\right)^{2} \\
C_{r}^{f} & \equiv 2 \Delta r_{-1}^{f} \Delta r_{-2}^{f} \\
D_{r}^{f} & \equiv-\left[\Delta r_{-1}^{f}+\Delta r_{-2}^{f}\right]^{2} \\
E_{r}^{f} & \equiv\left(r^{f}\right)^{2} \Delta r_{-2}^{f}-\left(r_{-1}^{f}\right)^{2}\left[\Delta r_{-1}^{f}+\Delta r_{-2}^{f}\right]+\left(r_{-2}^{f}\right)^{2} \Delta r_{-1}^{f}
\end{aligned}
$$

with $r \in[x, y, z]$.

Inserting (16)-(23) into the radiative transfer Eq. (14), we obtain

$$
\begin{aligned}
& \frac{\sin \vartheta_{g} \cos \varphi_{g}}{E_{x}^{f}}\left[\left(C_{x}^{f}+A_{x}^{f}\right) I_{0,0,0}^{f, g}+D_{x}^{f} I_{-1,0,0}^{f, g}+B_{x}^{f} I_{-2,0,0}^{f, g}\right] \\
& +\frac{\sin \vartheta_{g} \sin \varphi_{g}}{E_{y}^{f}}\left[\left(C_{y}^{f}+A_{y}^{f}\right) I_{0,0,0}^{f, g}+D_{y}^{f} I_{0,-1,0}^{f, g}+B_{y}^{f} I_{0,-2,0}^{f, g}\right] \\
& +\frac{\cos \vartheta_{g}^{g}}{E_{z}^{f}}\left[\left(C_{z}^{f}+A_{z}^{f}\right) I_{0,0,0}^{f, g}+D_{z}^{f} I_{0,0,-1}^{f, g}+B_{z}^{f} I_{0,0,-2}^{f, g}\right]= \\
& -\kappa_{0,0,0}^{\mathrm{ext}, f} I_{0,0,0}^{f, g}+S_{0,0,0}^{f, g} .
\end{aligned}
$$

The known factors can be abbreviated as

$$
\begin{aligned}
\alpha_{x}^{f, g} & \equiv \frac{\sin \vartheta_{g} \cos \varphi_{g}}{E_{x}^{f}} \\
\beta_{y}^{f, g} & \equiv \frac{\sin \vartheta_{g} \sin \varphi_{g}}{E_{y}^{f}} \\
\gamma_{z}^{f, g} & \equiv \frac{\cos \vartheta_{g}}{E_{z}^{f}}
\end{aligned}
$$

and solving the equation system with respect to $I_{0,0,0}^{f, g}$ yields

$$
\begin{aligned}
I_{0,0,0}^{f, g}= & \frac{U_{0,0,0}^{f, g}}{V_{0,0,0}^{f, g}} \\
V_{0,0,0}^{f, g} \equiv & \kappa_{0,0,0}^{\mathrm{ext}, f}+\alpha_{x}^{f, g}\left(C_{x}^{f}+A_{x}^{f}\right)+\beta_{y}^{f, g}\left(C_{y}^{f}+A_{y}^{f}\right) \\
& +\gamma_{z}^{f, g}\left(C_{z}^{f}+A_{z}^{f}\right) \\
U_{0,0,0}^{f, g} \equiv & S_{0,0,0}^{f, g}-\alpha_{x}^{f, g}\left(D_{x}^{f} I_{-1,0,0}^{f, g}+B_{x}^{f} I_{-2,0,0}^{f, g}\right) \\
& -\beta_{y}^{f, g}\left(D_{y}^{f} I_{0,-1,0}^{f, g}+B_{y}^{f} I_{0,-2,0}^{f, g}\right) \\
& -\gamma_{z}^{f, g}\left(D_{z}^{f} I_{0,0,-1}^{f, g}+B_{z}^{f} I_{0,0,-2}^{f, g}\right) .
\end{aligned}
$$

With (28), we can calculate the intensity of the scattered light at any grid point when the intensities at the previous two grid points along the three Cartesian axis are known. The density at the points of the adaptive grid is contained in $\kappa_{0,0,0}^{\text {ext } f}$ and $S_{0,0,0}^{f, g}$.

The boundary values are defined on a shadow grid that is not part of the adaptive photon grid. The boundary intensities are determined by the interstellar radiation field and nearby sources. As we use a 2 nd order differencing scheme, the shadow grid will be 2 cells deep. Figure $5 b$ shows such a shadow grid for a ray direction with positive components indicated by the arrow in the bottom right corner. The cell size of the shadow grid will not influence the solution and is chosen to be $1 / 10$ of the computational domain.

Since intensities of radiation propagating in all directions are calculated, the stencil will also be moved through the grid 


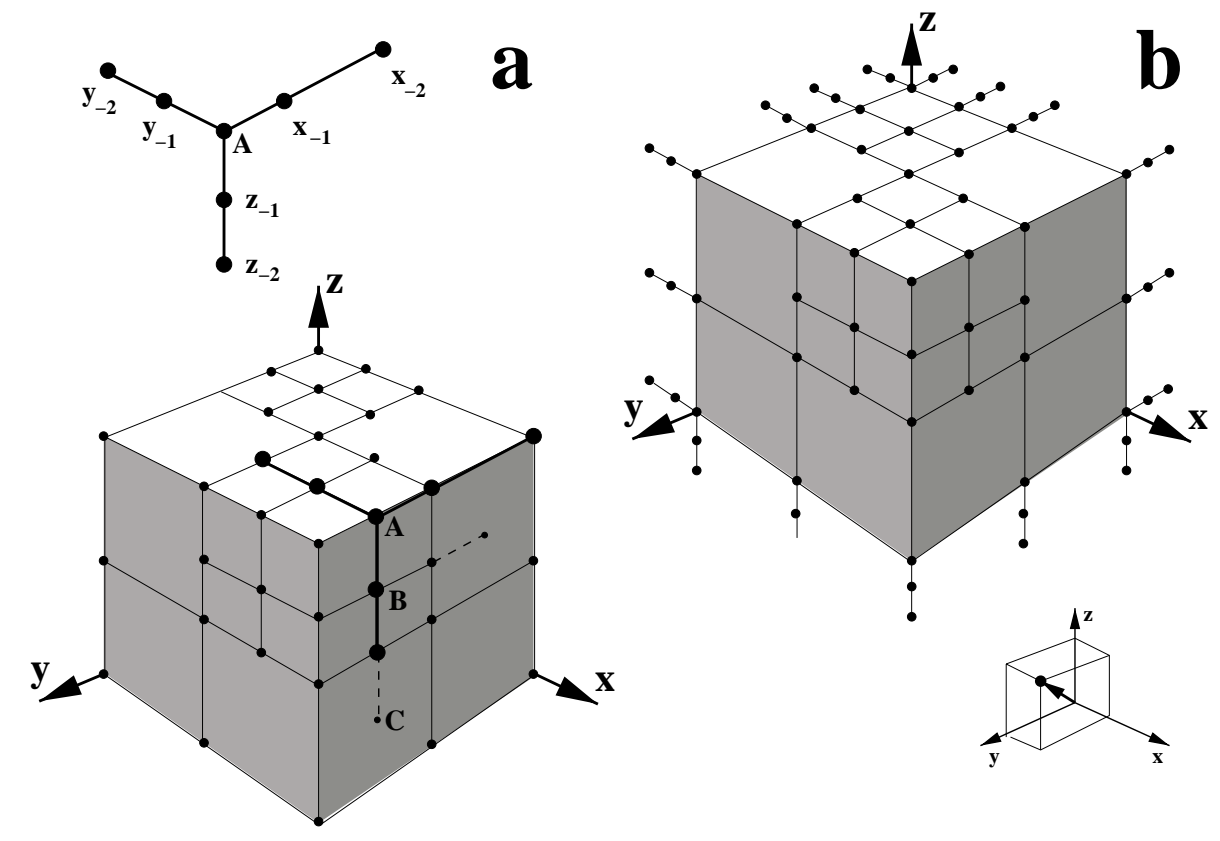

Fig. 5. Panel a) Sketch of a refined grid and the location of the seven point 2nd order finite differencing stencil centered at point A. In the upper part, the points are labeled according to their numbering with respect to point $\mathrm{A}$ along the $x, y$, and $z$-direction, respectively. Point $\mathrm{B}$ marks the center of a stencil moved downward with respect to A, with the point $\mathrm{C}$ indicating a stencil point which is not located on the grid. Panel $\mathbf{b}$ ) Refined grid and two cell deep shadow grid for a direction that is indicated in the small coordinate system at the bottom.

in other directions than the one following the orientation of the axis. Equation (28) is also valid also for the down-winding case (one of the components of direction vector $\boldsymbol{n}$ is negative) if you re-order the grid points with respect to the direction vector $\boldsymbol{n}$, as can be shown by considering $r_{0}^{f}<r_{-1}^{f}<r_{-2}^{f}$ with $r \in[x, y, z]$.

A major numerical concern in $3 \mathrm{D}$ calculations is to reduce the amount of calculations within the innermost loop of the RT solver as much as possible. In this loop, (7) and (8) are solved by $\Lambda$-iteration. We therefore pre-calculate the positions of all stencils for all directions. There are eight different direction types to be considered ranging from all three Cartesian components of $\boldsymbol{n}$ being positive to all being negative. Beside that, the standard refinement used in generating the grids guarantees that we can switch from a floating point storage of the grid points to integer values as multiples of the smallest occurring grid cell size. This reduces storage space of the grids and increases the speed of the solution substantially.

Note that the solution scheme (28) has been derived for a Cartesian grid, but equivalent schemes could be derived for other coordinate systems.

The overall solution strategy can be summarized as follows: we specify a density distribution of the dust, its size distribution, absorption and scattering properties, as well as the scattering phase function. We define the location and the spectrum of the radiation sources, and the boundary conditions. We specify the limiting wavelength for which we can neglect scattering.

To start the computation, we analyze the density distribution and determine minima, maxima and strong gradients. Using the concept of penetration depths (see Steinacker et al. 2002b), we construct adaptive photon transport grids for each wavelength. For the temperature, we create another grid depending on the symmetry of the density and source distribution.
On this grid, we determine a start temperature by illuminating each cell by the sources and then each cell by each cell. With this temperature $T_{0}$, the unprocessed intensity $I_{*}$ is calculated by solving (7). If scattering is important, also $I$ is determined by solving (8). In the latter case, we start with the scattered intensity from the formerly treated wavelength and calculate the source term (6). Then, the intensity is updated solving (8) with this source term and this $\Lambda$-iteration is continued until the intensity change drops below a given value $\chi$ (typically we choose $\chi=10^{-2}$ ). We use the standard technique proposed in $\mathrm{Ng}$ (1974) to accelerate convergence by using former solutions.

When $I_{\text {tot }}$ has been calculated, we update the temperature using (9), and continue this outer $\Lambda$-iteration until the temperature has converged to the self-consistent value. During the iterations, we check the global energy to be conserved (and equal to the start source energy).

\section{Discretization of the temperature distribution}

It is possible to derive a priori guesses as to where the radiation transport needs fine spatial grids to resolve the physics correctly and hence to deduce the emerging radiation. Knowing the density distribution and the optical properties of the dust particles, the optical depth throughout a grid cell can be used to derive a wavelength-dependent first order finite differencing criterion for the grid generation (see Steinacker et al. 2002b). In most cases, this leads to a refinement in the region around $\tau=1$. For the temperature, this is hardly possible, as the temperature couples to all wavelengths via (9) and hence, a clear definition of the $\tau=1$ layer is not evident. In regions where the radiation field is dominated by the source contribution, the temperatures are expected to be high with high 

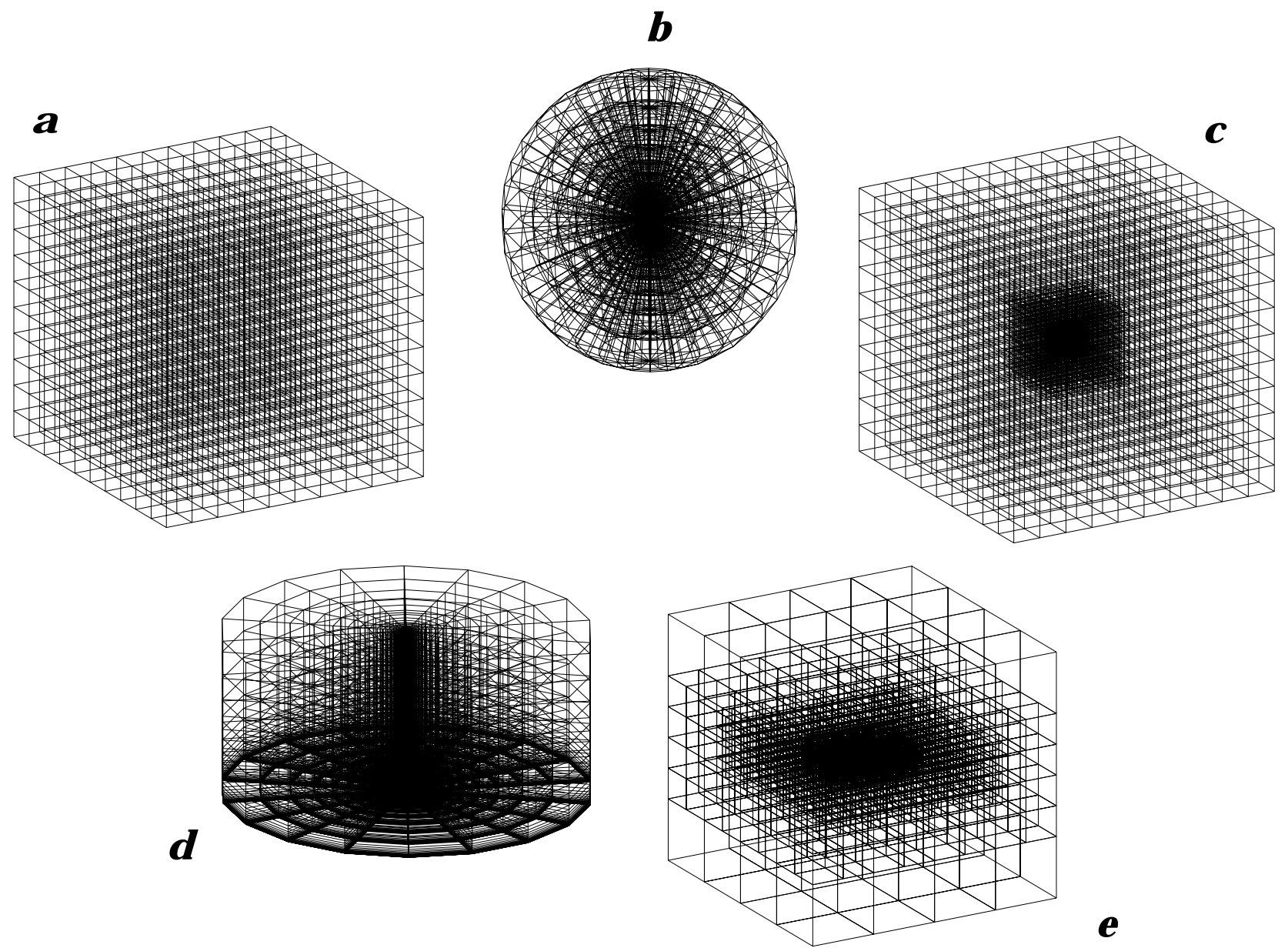

Fig. 6. Examples of temperature grids used by SteinRay. Depending on the density distribution, equidistant Cartesian a), spherical grids with logarithmic radial points b), strongly overlapping nested Cartesian grids $\mathbf{c}$ ), cylindrical grids with logarithmic height griding d), and adaptive grids with standard refinement $\mathbf{e}$ ) are used. The number of grid points has been reduced to keep the figure readable.

gradients. Considering e.g. an accretion disk, strong temperature gradients at the inner boundary and in the disk atmosphere are related to strong density gradients. For shielded regions, radiation is dominated by re-emitted long-wavelength dust emission and the temperature gradients should be small.

Therefore, adaptively refining grids similar to the density grids obtained in Steinacker et al. (2002b) could be used providing a global error control also for the temperature. However, the use of two different adaptive grids is very time-consuming. It has to be kept in mind that both the ray-tracing solver and the scattering-integral solver on the photon grids need the temperature at arbitrary points within the computational domain, and this very often. An 8-point interpolation is an acceptable effort, but with an adaptive grid, a tree-code search algorithm is needed to find the corresponding grid cell, which is too much of an effort especially for the scattering integral solver. In view of the expected smooth behavior (compared to the radiation field) of the temperature, simpler grids are used in STEINRAY with the drawback of having no a-priori error control for the temperature. Some of them are plotted in Fig. 6, namely an equidistant Cartesian grid (a), a spherical grid with radial points being logarithmically equidistant (b), strongly overlapping nested Cartesian grids (c), cylindrical grids with logarithmic height griding (d), and adaptive grids with standard refinement (e) for cases where the same grid for radiation and temperature can be used as the gradients within the object are smooth.

The outer temperature iteration requires to start with a given initial temperature distribution $T_{0}(\boldsymbol{x})$ and then to improve this distribution using (9) to obtain the correct temperatures $T(\boldsymbol{x})$. As this outer iteration requires to run the entire solution process several times, it is essential to have quick convergence and a good initial guess. In the Appendix, we derive a scheme to calculate the initial temperature distribution $T_{0}$ for a given density distribution and optical dust properties. $T_{0}$ is obtained neglecting scattering but considering both stellar-cell and cell-cell illumination. For deeply embedded sources, the transformation of short-wavelength photons to infrared radiation takes place in a thin dust layer, and as it matters little for the overall temperature if the radiation is scattered there a few times, $T-T_{0}$ will be small and convergence fast. If one deals with a configuration that has an extended atmosphere, namely a region that can be seen from outside with an optical depth of the order of 1 for wavelengths where scattering is important (like e.g. an accretion disk), $T_{0}$ and $T$ will differ substantially in visible regions and thus the images calculated from them will deviate too. As we have pointed out in Sect. 3, forward 
scattering dominates the scattering processes at wavelengths of strong scattering. An improved $T_{1}$ can be calculated using this effect: in the source illumination of each cell, only the absorption instead of the complete extinction is considered, arguing that the radiation is not nearly lost but scattered forward in first approximation.

\section{Applications}

Testing RT codes is difficult as even in simple 1D cases, for realistic density distributions, the RT equation already gets too complicated to be solved analytically. Benchmark studies based on well-defined RT problems can aid here to compare the results of different codes. It may be pointed out here explicitly, though, that there may be situations where the majority of codes fails, e.g. due to resolution problems of standard algorithms. Only by incorporating error control is it possible to infer reliability of the technique. Hence, error control in STEINRAY will be one of the issues we will discuss in the following.

For 1D continuum RT, a benchmark was published by Ivezic et al. (1997). They compared results of three codes with similar algorithms for an application in spherical geometry. For 2D continuum RT, Pascucci et al. (2003) have defined a benchmark based on simulations of a standard accretion disk around a solar-type star. The project involved four separately developed codes, two based on the Monte-Carlo technique and two based on grids, each with very different implementation. SteinRay has been tested in this collaboration in good overall agreement with the other three codes.

There is no benchmark project for $3 \mathrm{D}$ continuum radiative transfer yet. Hence, we present first results of the code twofolded: we show simple test cases where the solution is either exactly or approximately known, and a more complex 3D scenario to show the capabilities. For applications to circumbinary disks, warped disks, disks with planets, and AGN dust tori, as well for a 3D benchmark test including images, we refer to the later papers of this series.

\subsection{Simple 1D test cases: Slab and spherically symmetric geometry}

In order to compare with an analytical solution, we start with the most simple case possible, namely radiation passing through a homogeneous dust medium with a constant density and no dust emission radiation. We follow the radiation up to a distance of $1000 \mathrm{AU}$ from the source that is emitting radiation just along the $x$-axis. For this so-called slab geometry, we assume a constant dust number density of $1 \mathrm{~m}^{-3}$, spherical silicate dust particles of size $0.12 \mu \mathrm{m}$, and a wavelength of $30 \mu \mathrm{m}$ so that scattering can be neglected. The optical depth

$\tau(\lambda, x)=\int_{0}^{x} \mathrm{~d} x^{\prime} \kappa\left(\lambda, x^{\prime}\right)$

along a ray (assumed to be parallel to the $x$-axis) simplifies in this case to $\tau(\lambda, x)=\kappa(\lambda) x$. The solution of the continuum RT Eq. (1) then is $\mathcal{I}(\lambda, x)=I_{0} \exp [-\tau(\lambda, x)]$ for all $\boldsymbol{x}$ and vanishes for all directions except along the $x$-axis $\boldsymbol{n}=\boldsymbol{e}_{x}$. Comparing

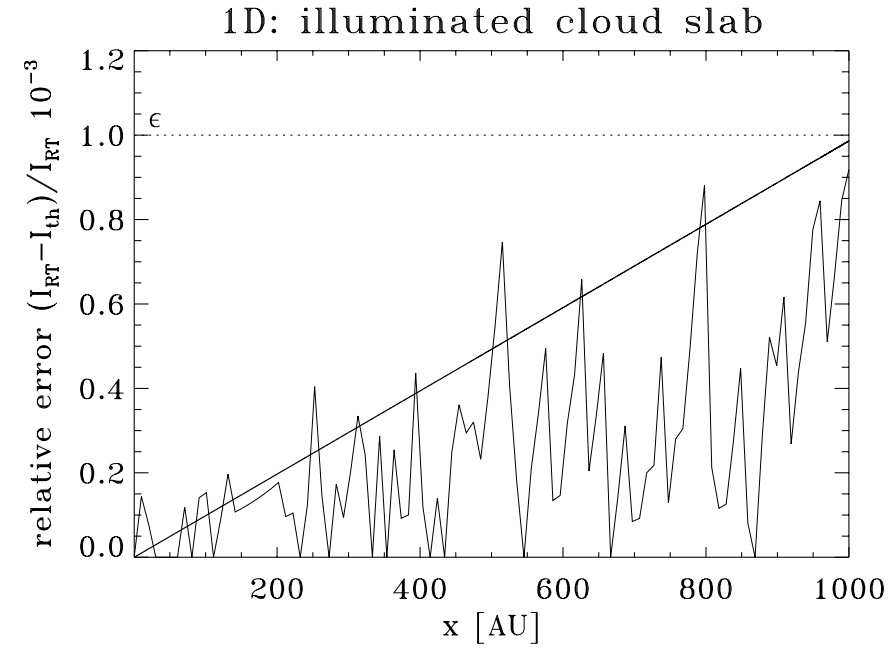

Fig. 7. Relative error of the intensity in a homogenous illuminated cloud slab without continuum re-emission of the dust. The straight line shows the error when using a constant stepsize tracer, the noisy line gives the error of a 5th-order Runge Kutta-solver.

the calculated intensity $I_{\mathrm{RT}}$ with the analytical solution $I_{\text {th }}$, we can analyze the behavior of the used ray-tracer. In Fig. 7, we show the relative error $\eta=\left(I_{\mathrm{RT}}-I_{\mathrm{th}}\right) / I_{\mathrm{RT}}$ in units of $10^{-3}$ as a function of $x$ for a tracing with constant steps (straight line) and with a 5th-order Runge Kutta algorithm and adaptive step size control (noisy line). Both solutions have errors below the given limit $\varepsilon=10^{-3}$. However, the adaptive step size solver will automatically use the lowest number of points possible, and works for any density distribution.

\subsection{Simple 1D test case: Spherically symmetric geometry}

In spherical geometry, the intensity will spatially depend on the radius only, and we can use the scaling argument by Ivezic et al. (1995) to reduce the amount of free parameters in the 1D RT problem by normalizing the occurring parameters. We use the benchmark provided by Ivezic et al. (1997) to test the code in spherical geometry. From their models, we choose the scaled example of a central source with $T_{*}=2500 \mathrm{~K}$, a temperature at the inner boundary of $T_{1}=800 \mathrm{~K}$, an outer radius normalized to the inner boundary of $Y=1000$, density power law index $p=0$ (constant density), and optical depth at the reference wavelength $\lambda_{1}=1 \mu \mathrm{m}$ to be $\tau_{1}=10$. For the sake of comparison, we also use isotropic scattering.

We show the adaptive photon transport grid for the scattered radiation at a wavelength of $\lambda=0.165 \mu \mathrm{m}$ in Fig. 8. As the penetration depth in this case is $d=473 \mathrm{AU}$, the grid generator has just refined the grid within a sphere of this radius to keep the optical depth across one grid cell below the given error limit of $\epsilon=10^{-2}$. The smallest cells are not plotted to keep the figure readable.

The upper panel of Fig. 9 shows the temperature we obtained (solid line) along the $z$-axis as a function of the normalized radius and the benchmark results (crosses). We confirm their findings and the agreement was achieved after 


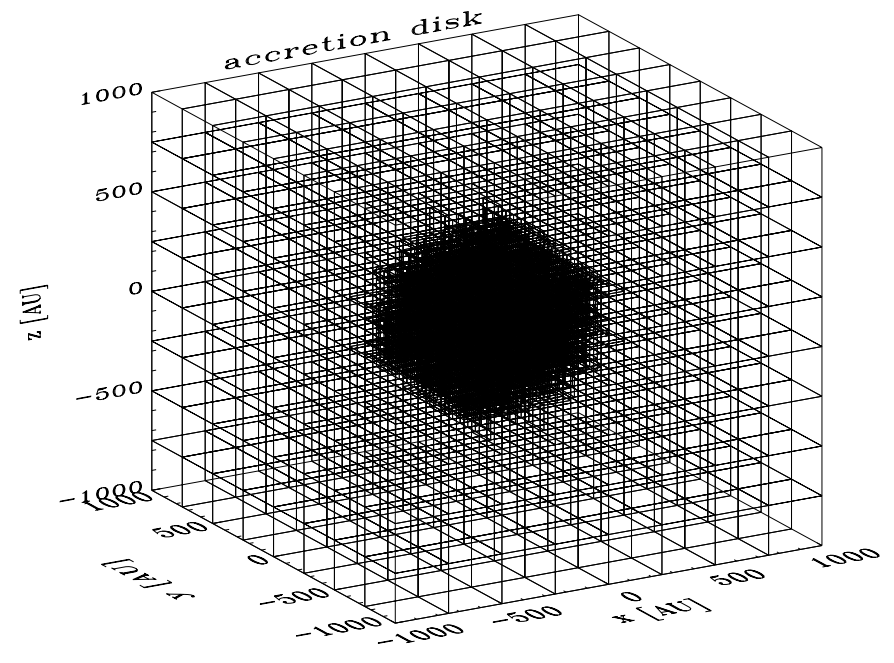

Fig. 8. Adaptive photon transport grid for the scattered part of the radiation passing through a dust envelope with spherically symmetric density distribution, for the wavelength $\lambda=0.165 \mu \mathrm{m}$.

three outer $\Lambda$-iterations. The deviation of the temperatures at a given radius for all directions in the spherical temperature grid was less than $1 \%$. In the lower panel of Fig. 9, the normalized, distance- and luminosity-independent spectral energy distribution $\lambda F_{\lambda} / \int F_{\lambda} \mathrm{d} \lambda$ is given as a function of the wavelength. Again, there is good agreement between benchmark result (crosses) and SteinRay simulation (solid line). The slight deviations might arise from the different resolution of the grids. Using the full symmetry of the problem, the 1D codes are able to spend all the computer resources on resolving 1 dimension, while the $3 \mathrm{D}$ code has to use a rather coarse grid of just $N_{\lambda}=60$ wavelength grid points, a direction grid of $N_{\theta} \phi=144$ for the scattered radiation, a spatial grid containing less than $10^{5}$ cells per wavelength, and temperature cell numbers fewer than $10^{6}$ for the entire domain.

\subsection{Full 3D test case: Two stars within cavities in a molecular cloud core}

Tests with a 2D RT benchmark have been given in Pascucci et al. (2003) comparing the results obtained with STEINRAY for a 2D standard accretion disk with three other 2D and 3D RT codes. They show very good overall agreement in both the spectrum and the temperature distribution. We therefore proceed to full 3D RT. But here, no benchmark has been defined sofar. And moreover, there is no benchmark yet that gives images for multi-dimensional continuum RT. So, to show the capabilities of the code, we just give here images of a complex test case and refer to further applications and benchmark runs in forthcoming papers.

The example we choose is a spherically symmetric cloud core that contains two young stars having formed at slightly different times, close to the core center. We assume the stars to be massive $\left(M=8 M_{\odot}\right)$, with a luminosity of $L=2200 L_{\odot}$, and a surface temperature of $T=20000 \mathrm{~K}$. The emerging radiation is assumed to have already formed cavities free of dust, and all remnants like disks that may have formed the stars
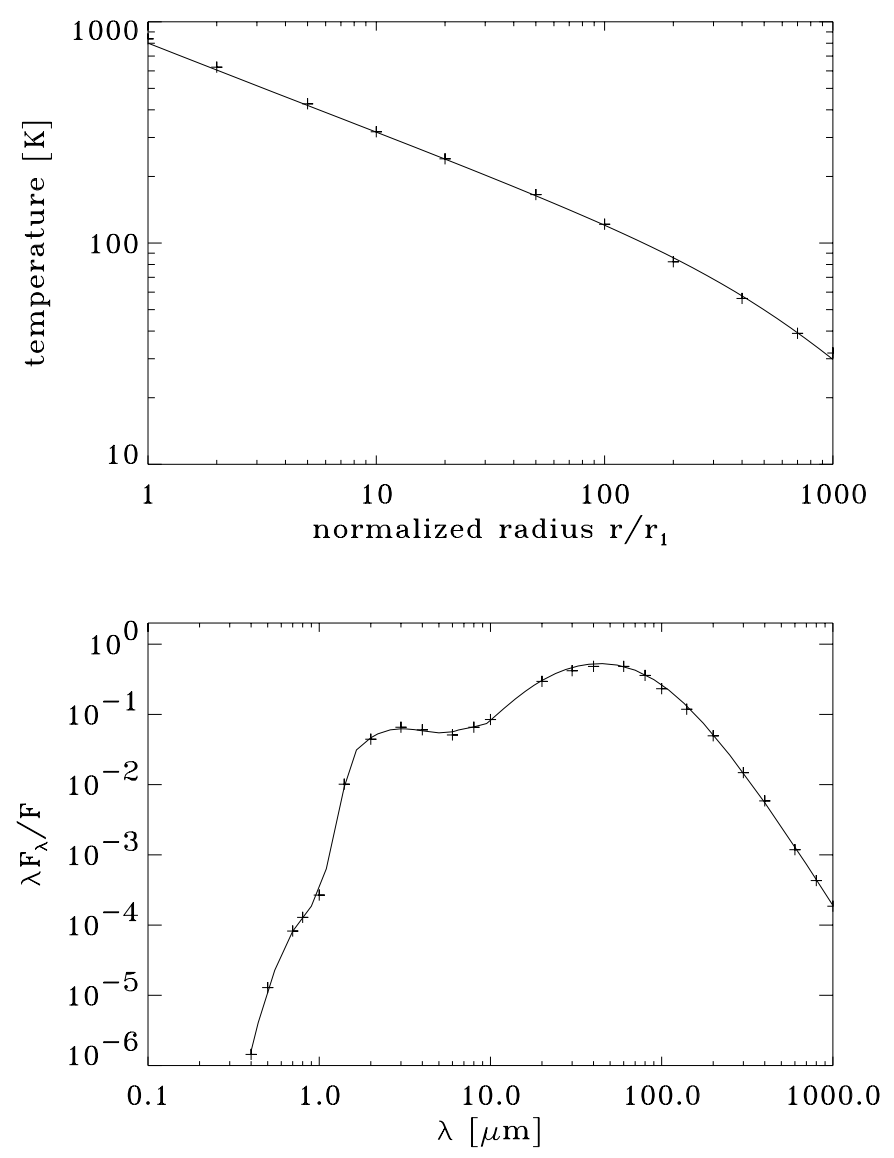

Fig. 9. Comparison with the 1D benchmark results of Ivezic et al. (1997) for $T_{1}=800 \mathrm{~K}, Y=1000, p=0, \tau_{1}=10, \lambda_{1}=1 \mu \mathrm{m}$, and a resulting ratio $\Psi=3$ of incoming and re-radiated flux at the inner boundary. The upper panel shows the benchmark temperatures as a function of normalized radius (crosses) and the result obtained with SteinRay (solid line). In the lower panel, the dimensionless spectral shape $\lambda F_{\lambda} / \int F_{\lambda} \mathrm{d} \lambda(\lambda)$ is plotted in the same notation.

are destroyed. We choose a slight time shift in the evolution to have cavities with different sizes $1000 \mathrm{AU}$ and $2000 \mathrm{AU}$, respectively, and at distances of 1500 and $2000 \mathrm{AU}$ from the core center. The stars are moved away from the cavity centers towards the core center to distances of $1500 \mathrm{AU}$ and $1000 \mathrm{AU}$, respectively. This is to take into account the density gradient within the core and its influence on the cavity formation. The density within the core is assumed to be a Gaussian of the form $\rho=\rho_{0} \exp \left[-r^{2} / r_{0}^{2}\right]$ with $\rho_{0}=1 \mathrm{~m}^{-3}$ and $r_{0}=2000 \mathrm{AU}$.

Figure 10 illustrates the density distribution in the $z=0$ plane. The position of the two stars is indicated by two peaks within the cavities. We consider this scenario as a possible snapshot from the evolution of a dense core where two stars have been formed and do not consider how stable this configuration will be over longer time scales (a distortion of the core center due to gravitational torque from the stars seems likely). With this strongly asymmetric configuration, the stars displaced from the center of the cavities of different size, and the inner core center between the stars, clearly, 3D RT is needed to calculate the self-consistent temperature distribution and to produce images for all wavelengths of interest. On the other side, the configuration is still simple enough to allow for 


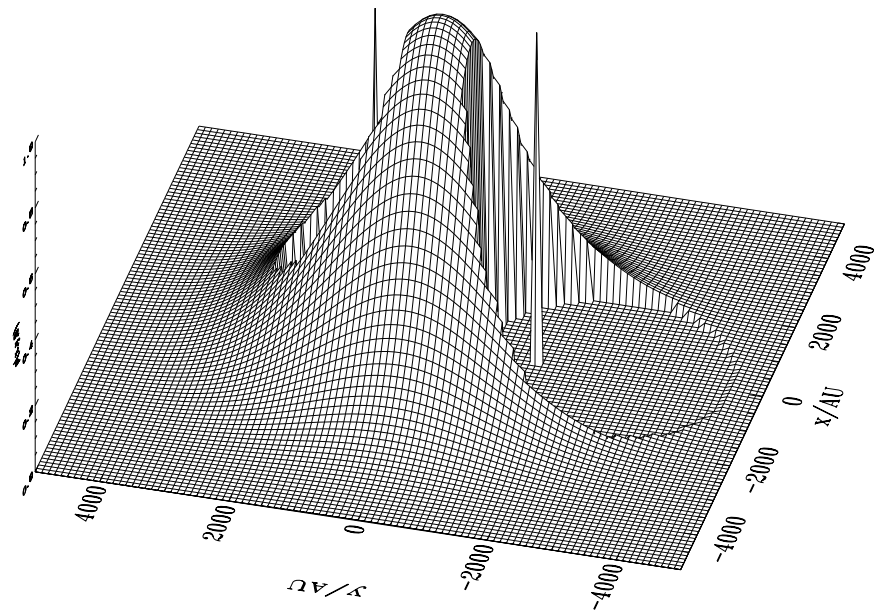

Fig. 10. Cut through the density distribution of the 3D test case at $z=0$. The two massive stars are located in cavities that have formed within a dense core with a density maximum in-between the two stars, their position is indicated by peaks.

interpretation of the obtained temperatures and images. The calculated self-consistent temperature in the $x$ - $y$-plane is shown in Fig. 11. In panel a, we show the temperature distribution for a run where we placed just one star on the $x$-axis at $x=$ $-1500 \mathrm{AU}$. The star heats the dust on its side of the core outside the cavity. To heat up the opposite side of the core, its radiation has to pass the inner core maximum and heating therefore is reduced. Radiation passing through the small empty cavity, though, can reach the dust behind the smaller cavity more easily than radiation not crossing the cavity. Therefore, the dust directly behind the smaller cavity should be hotter than dust that is heated by radiation not passing through the smaller cavity. This effect can indeed be seen in panel a. Note that scattering blurs this effect as for $\lambda<6 \mu \mathrm{m}$, radiation is scattered out or into the cavities. In panel $b$, we give the temperature distribution when the other star is added to the smaller cavity. Due to the smaller extent of the small cavity, it is not able to heat the material in the vicinity of the cavity to higher temperatures than the star in the larger cavity, as the optical depth is higher. In Fig. 12, we show the images of the cavity configuration at wavelengths of $\lambda=3 \mu \mathrm{m}, 10 \mu \mathrm{m}, 20 \mu \mathrm{m}$, and $250 \mu \mathrm{m}$, respectively. The view is inclined by $50^{\circ}$ with respect to the $x$-axis where both stars are located, and the smaller cavity is in the front.

For $\lambda=250 \mu \mathrm{m}$, the configuration is optically thin and the dust emission at the surface of both cavity shells accumulates so that they are clearly visible at all viewing angles. The reemission function

$R \equiv \kappa^{\mathrm{abs}}(\lambda, \boldsymbol{x}) B[\lambda, T(\boldsymbol{x})]$

of the dust has its maximum for temperatures around $15 \mathrm{~K}$, so emission should mainly be expected from the outer parts. But due to the gradient of the Gaussian profile of the dense core, radiation of the hotter dust at the surface of the cavities still dominates, which radiates according to $R$ with Planck emission in the Rayleigh-Jeans range. The stars do not emit substantially at this wavelength.
At wavelength of $\lambda=20 \mu \mathrm{m}$, the inner part of the core is optically thick. Part of the emission from the dust around the larger cavity is obscured by the dense core. The same is valid for the image at $\lambda=10 \mu \mathrm{m}$. As the absorption is stronger here, the smaller cavity is deeply embedded and emission is only seen from parts where the density of the dense core drop due to the Gaussian profile. In both pictures, emission from the star is too weak to be seen.

In the left upper panel of Fig. 12, the stars can be seen at $\lambda=3 \mu \mathrm{m}$ (view along the $y$-axis, stars at $x=1000 \mathrm{AU}$ and $-1500 \mathrm{AU}$, total extend $10000 \mathrm{AU})$. Still it is the dust close to the surface of the cavity that is dominating the emission, but only the emission in the outer parts can be seen as the extinction drops with the density.

The emission from the cavity surfaces is not entirely smooth, showing a slight numerically caused ring pattern. This is an effect of the finite grid size of $100 \mathrm{AU}$ of the temperature grid. Doubling the number of grid points in each direction (100 to 200) would increase the size of the temperature array by a factor of 8 and the computational time by a factor of 64 . The use of an adaptive temperature grid reduces the finite grid cell errors but increases the runtime as the interpolation between the adaptive photon transport grid for the scattered radiation and the temperature grid becomes more time-consuming.

The numerically by far most difficult part is to produce images in the optical and UV. We have chosen $\lambda=0.344 \mu \mathrm{m}$, as the scattering is maximal in this wavelength range. With a deeply embedded pair of stars like in our configuration, hardly any scattered light can be expected to leave the core before being absorbed. As the images showed just the reddened stars, we decided to lower the density by a factor of 50 to make escape of scattered light possible. We show two images at this wavelength in Fig. 13 with an inclination of the view by $30^{\circ}$ with respect to the $x$-axis, and with the large cavity in the front. The left image gives the intensity of scattered radiation calculated under the assumption of isotropic scattering. Most emission comes from the radiation scattered in the cavity. Its ringlike shape is an interplay of the low density in the right part of the cavity reducing scattering and the high density towards the center causing absorption. There is also weak diffuse scattering in the entire core with radial gradient following the density gradient.

The right image is calculated for the same viewing angle and wavelength, but with a realistic scattering function as described in Sect. 3. At $\lambda=0.344 \mu \mathrm{m}$, forward scattering dominates. Therefore, only the front part of the surface of the larger cavity that is close to the star and that has substantial density can scatter the radiation to the observer. Additionally there is some diffuse scattered radiation coming from the front border of the dense center of the core caused by the other star.

This comparison shows that a wrong approximation of the scattering will alter the resulting images substantially. Animations varying the viewing angle to support visualization of the 3D structure can be found under http://www . astro. uni-jena.de/Users/stein/Ani/anin.htm.

Both ray-tracing and finite differencing on the transport grids allow error control of the solution. Nevertheless, we point out that in contrast to the intensity calculations, for the 

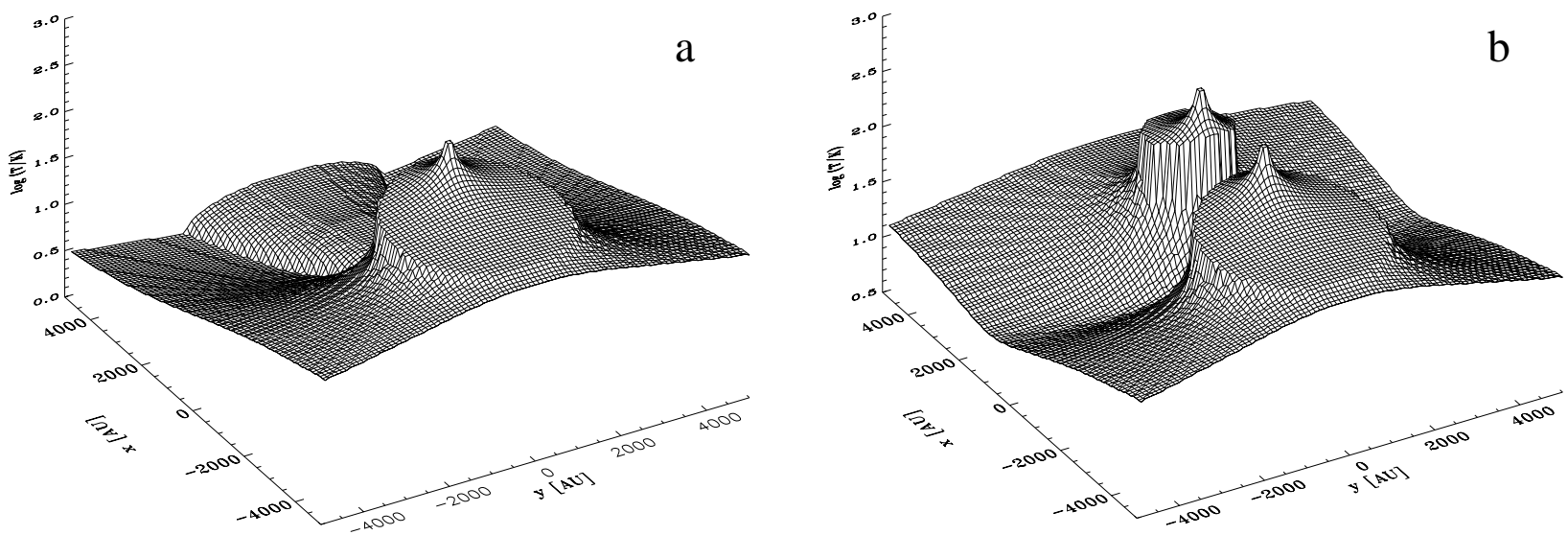

Fig. 11. Temperature distribution of the cavity configuration in the $x$-y-plane. a) Single star located on the $x$-axis at $x=-1500$ AU. b) Two stars located on the $x$-axis at $x=1000$ AU and $x=-1500$ AU.
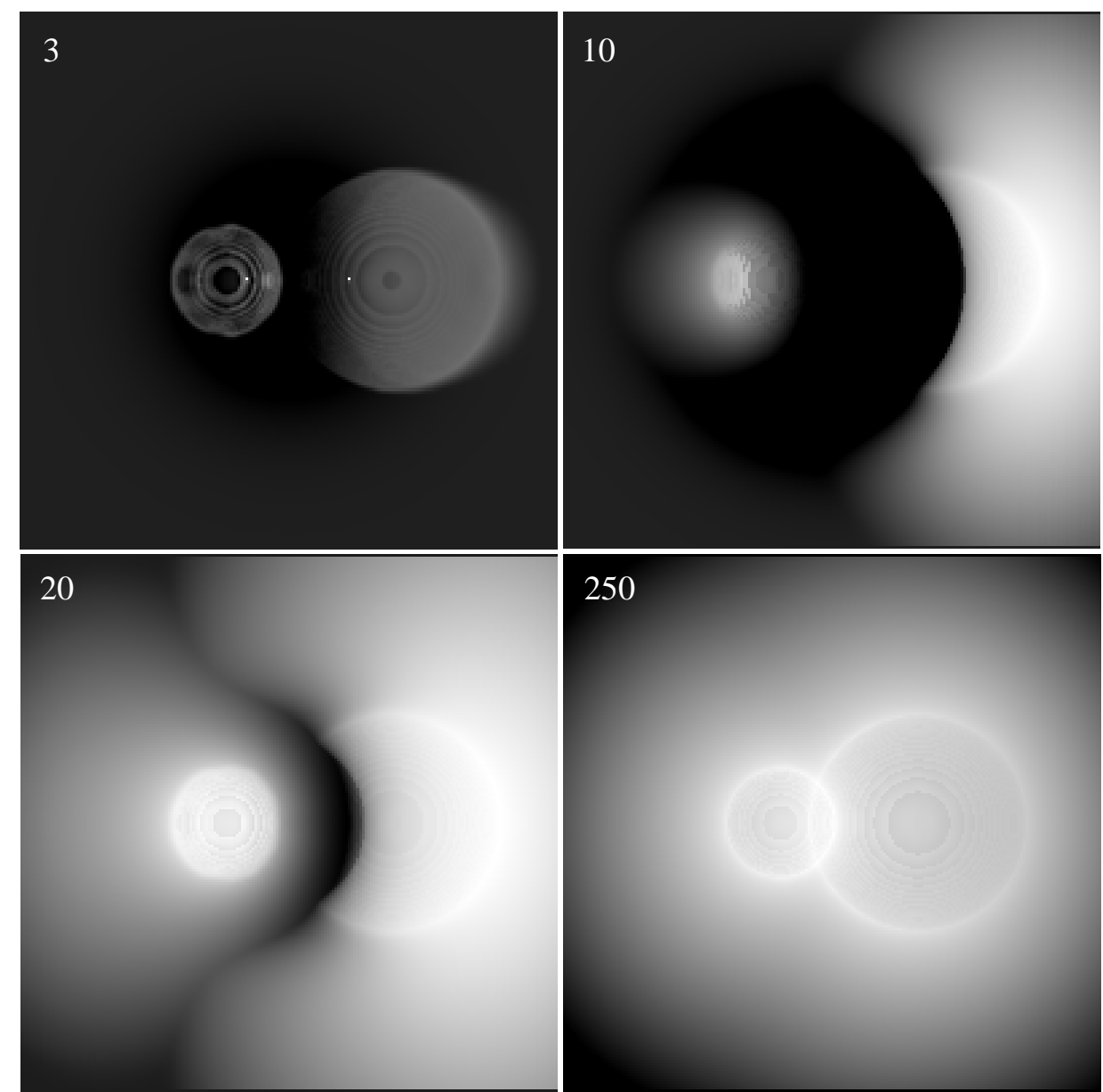

Fig. 12. Images of the cavity configuration at $\lambda=3 \mu \mathrm{m}, 10 \mu \mathrm{m}, 20 \mu \mathrm{m}$, and $250 \mu \mathrm{m}$, respectively. The stars are located at $x=1000$ AU and $-1500 \mathrm{AU}$ on the $x$-axis, the viewing angle is inclined by $50^{\circ}$ so that the smaller cavity is in the front.

temperature iteration, it is impossible to give an a priori error limit. Here, we rely on usual a posteriori methods, namely to check the global energy conservation when integrating the outcoming flux over a closed surface around the source of energy. The main sources of numerical errors are the interpolations of the temperatures for the ray-tracing and the transport grids. As they have to be carried out often, a quadratic or higher polynomial interpolation is too time-consuming. The same is valid for temperature grids with cell numbers exceeding $10^{6}$. For the simulations, global energy conservation within 5\% was used as convergence criterion for the outer $\Lambda$-iteration.

\section{Conclusions}

In this paper, we presented the solution algorithm implemented in the grid-based code SteinRay, designed to solve the 3D 

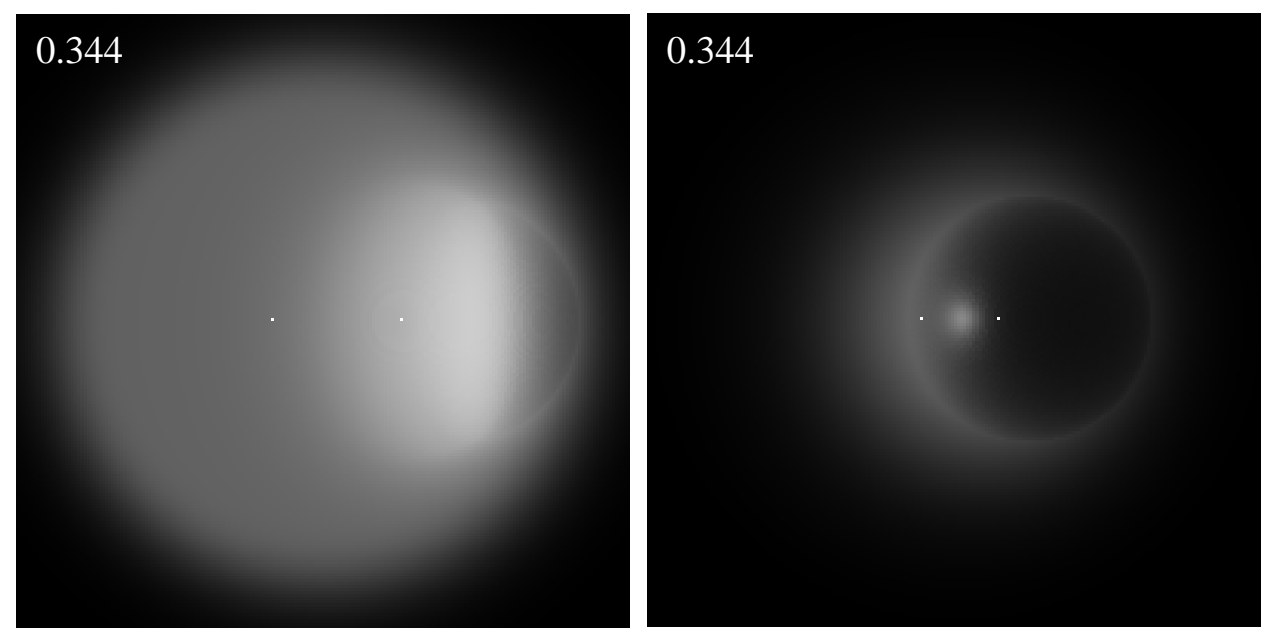

Fig. 13. Images of the cavity configuration at $\lambda=0.344 \mu \mathrm{m}$. The stars are located at $x=1000 \mathrm{AU}$ and $-1500 \mathrm{AU}$ on the $x$-axis, the viewing angle is inclined by $20^{\circ}$ so that the larger cavity is in the front. The left image has been obtained using isotropic scattering, the right image has been calculated with the correct phase function.

continuum radiative transfer problem for the intensity emerging from objects in star-forming regions, evolved stars with envelopes, starburst galaxies, and AGNs. The method is a combination of step-size controlled ray-tracing and solution on adaptive multi-wavelength photon transport grids, on which the finite-differencing discretization error is minimized. We briefly analyzed the optical properties of typical cosmic dust grains, and discussed the wavelength range for which the timeconsuming solution on adaptive grids has to be used. For the temperature, we have presented and discussed possible grids on which the temperature distribution can be calculated selfconsistently. Aside from 2D benchmark comparisons presented elsewhere, we have tested the code with simple 1D cases and illustrated the capabilities by treating a complex 3D test case. The temperature is calculated self-consistently using standard accelerated $\Lambda$-iteration.

Acknowledgements. J. S. thanks F. Evans for valuable comments in the course of improving the adaptive photon transport grids. We thank D. Folini for an excellent referee report helping to improve the paper at various points.

\section{Appendix: Initial temperature distribution}

Computer time can be saved substantially when starting the solution of the RT equation with a temperature that is already close to the correct value. The temperature iteration in the RT solution scheme will converge quickly when an initial temperature is used that is derived for the case that the high-energetic source radiation has been absorbed and re-distributed by the dust particles already. Here, we derive a scheme to calculate the initial temperature for a given dust configuration. The main approximation is that the influence of scattering is neglected for the heating process. In view of the strong forward scattering in the wavelength regions where scattering plays a role, this assumption is reasonable to derive an initial temperature distribution. We concentrate here on stellar illumination, but a corresponding scheme can be derived for other radiation sources like evolved stars or the central engine of an active galactic nucleus.
The power emitted by a star with the luminosity $\mathcal{L}_{\lambda}^{*}$ that is received by the dust particle $k$ of size $a$ with the absorption efficiency $Q_{\lambda}^{\text {abs }}$ at a distance $r_{k}$ through vacuum is (Evans 1994)

$$
P_{\mathrm{in}}^{*}(k)=\frac{\pi a^{2}}{4 \pi r_{k}^{2}} \int_{0}^{\infty} \mathrm{d} \lambda \mathcal{L}_{\lambda}^{*} Q_{\lambda}^{\mathrm{abs}} .
$$

With $R_{*}$ and $T_{*}$ being the stellar radius and effective surface temperature, respectively, the luminosity is $\mathcal{L}_{\lambda}^{*}=4 \pi R_{*}^{2} \pi B_{\lambda}\left(T_{*}\right)$ and we get

$$
P_{\mathrm{in}}^{*}(k)=\pi a^{2}\left(\frac{R_{*}}{r_{k}}\right)^{2} \int_{0}^{\infty} \mathrm{d} \lambda \pi B_{\lambda}\left(T_{*}\right) Q_{\lambda}^{\mathrm{abs}} .
$$

Dividing the domain into $N_{\mathrm{c}}$ cell cubes of equal density and temperature within the cube, with volume $V$, the number of particles $N_{K}$ in a cell $K$ can be obtained from the number density $n(\boldsymbol{x})$ using

$N_{K}=V_{K} n\left(x_{K}\right)$,

if a single-sized distribution of the dust is assumed. Hence, the stellar power received at cell $K$ writes as

$P_{\mathrm{in}}^{*}(K)=V_{K} n\left(\boldsymbol{x}_{K}\right) \pi a^{2}\left(\frac{R_{*}}{r_{* K}}\right)^{2} \int_{0}^{\infty} \mathrm{d} \lambda \pi B_{\lambda}\left(T_{*}\right) Q_{\lambda}^{\mathrm{abs}}$,

where $r_{* K}$ is the distance between the star and cell $K$.

In the presence of extincting matter between star and dust, the intensity along a ray $\boldsymbol{x}=u \boldsymbol{e}_{* K}$ from the star to cell $K$ is damped according to

$\frac{\mathrm{d} I_{\lambda}}{\mathrm{d} u}=-\pi a^{2} Q_{\lambda}^{\mathrm{ext}} n(u) I_{\lambda}(u)$

with the unit vector $\boldsymbol{e}_{* K}$ pointing from the star to cell $K$, yielding

$$
\begin{aligned}
I_{\lambda}\left(\boldsymbol{x}_{K}\right) & =B_{\lambda}\left(T_{*}\right) * \exp \left[-\pi a^{2} Q_{\lambda}^{\mathrm{ext}} \int_{0}^{u_{K}} \mathrm{~d} u^{\prime} n\left(u^{\prime}\right)\right] \\
& \equiv B_{\lambda}\left(T_{*}\right) D_{* K}^{\lambda}
\end{aligned}
$$


leaving the stellar power received at cell $K$ to be

$P_{\text {in }}^{*}(K)=V_{K} n\left(x_{K}\right) \pi a^{2}\left(\frac{R_{*}}{r_{* K}}\right)^{2} \int_{0}^{\infty} \mathrm{d} \lambda \pi B_{\lambda}\left(T_{*}\right) D_{* K}^{\lambda} Q_{\lambda}^{\text {abs }}$.

To define the temperature $T_{K}$, we consider the power re-emitted by the cell $K$ in local thermal equilibrium

$P_{\text {out }}(K)=V_{K} n\left(\boldsymbol{x}_{K}\right) 4 \pi a^{2} \int_{0}^{\infty} \mathrm{d} \lambda \pi B_{\lambda}\left(T_{K}\right) Q_{\lambda}^{\mathrm{abs}}$.

Hence, a starting temperature distribution $T^{0}$ for the cells can be obtained just from stellar illumination by setting $P_{\text {in }}^{*}(K)=$ $P_{\text {out }}(K)$ for each cell. Inserting we find

$$
\int_{0}^{\infty} \mathrm{d} \lambda B_{\lambda}\left(T_{K}^{0}\right) Q_{\lambda}^{\mathrm{abs}}=\frac{1}{4}\left(\frac{R_{*}}{r_{* K}}\right)^{2} \int_{0}^{\infty} \mathrm{d} \lambda B_{\lambda}\left(T_{*}\right) D_{* K}^{\lambda} Q_{\lambda}^{\mathrm{abs}} .
$$

In the next step, we consider that the cells illuminate each other. The power that is received by cell $L$ from cell $K$ is

$$
\begin{aligned}
P_{\mathrm{in}}^{K}(L)= & V_{L} n\left(\boldsymbol{x}_{L}\right) \frac{\pi a^{2}}{4 \pi r_{K L}^{2}} V_{K} n\left(\boldsymbol{x}_{K}\right) 4 \pi a^{2} \\
& \times \int_{0}^{\infty} \mathrm{d} \lambda \pi B_{\lambda}\left(T_{K}^{0}\right) D_{K L}^{\lambda} Q_{\lambda}^{\mathrm{abs}} \\
= & V_{L} V_{K} n\left(\boldsymbol{x}_{L}\right) n\left(\boldsymbol{x}_{K}\right) \frac{\pi a^{4}}{r_{K L}^{2}} \\
& \times \int_{0}^{\infty} \mathrm{d} \lambda \pi B_{\lambda}\left(T_{K}^{0}\right) D_{K L}^{\lambda} Q_{\lambda}^{\mathrm{abs}} .
\end{aligned}
$$

The total power received at cell $L$ therefore is

$$
P_{\text {in }}(L)=P_{\text {in }}^{*}(L)+\sum_{\substack{K=1 \\ K \neq L}}^{N c} P_{\text {in }}^{K}(L)
$$

The updated temperature $T_{L}^{1}$ yields from $P_{\text {in }}(L)=P_{\text {out }}(L)$ as

$$
\begin{gathered}
\int_{0}^{\infty} \mathrm{d} \lambda B_{\lambda}\left(T_{L}^{1}\right) Q_{\lambda}^{\mathrm{abs}}=\frac{1}{4}\left(\frac{R_{*}}{r_{* L}}\right)^{2} \int_{0}^{\infty} \mathrm{d} \lambda B_{\lambda}\left(T_{*}\right) D_{* L}^{\lambda} Q_{\lambda}^{\mathrm{abs}} \\
+\frac{1}{4} \sum_{\substack{K=1 \\
K \neq L}}^{N c} V_{K} n\left(\boldsymbol{x}_{K}\right) \frac{a^{2}}{r_{K L}^{2}} \int_{0}^{\infty} \mathrm{d} \lambda B_{\lambda}\left(T_{K}^{0}\right) D_{K L}^{\lambda} Q_{\lambda}^{\mathrm{abs}} .
\end{gathered}
$$

This formula applies also for the $i$ th iteration, where $T_{L}^{1}$ and $T_{L}^{0}$ are exchanged by $T_{L}^{i+1}$ and $T_{L}^{i}$, respectively.

Numerically, $D_{* L}^{\lambda}$ and $D_{K L}^{\lambda}$ are obtained from ray-tracing. For large numbers of cells, ray-tracing should be truncated when the intensity has dropped below a given limit characterizing a small fraction of the thermal energy in the cell. In the optically thick case, only cells located within a few mean free paths of the photons will contribute to the cell-cell illumination.

We calculate

$$
W_{K L}^{i, \lambda}=B_{\lambda}\left(T_{K}^{i}\right) D_{K L}^{\lambda} Q_{\lambda}^{\mathrm{abs}}
$$

on a given wavelength grid $\lambda_{f}$ for $f=1, \ldots, N_{\lambda}$ and use trapezoidal integration

$$
\int F(x) \mathrm{d} x \approx \frac{1}{2} \sum_{f=2}^{N}\left[F\left(x_{f}\right)+F\left(x_{f-1}\right)\right]\left(x_{f}-x_{f-1}\right) .
$$

The numerical scheme for calculating the temperature distribution iteratively is

$$
\begin{aligned}
& \sum_{f=2}^{N_{\lambda}}\left[B_{\lambda_{f}}\left(T_{L}^{i+1}\right) Q_{\lambda_{f}}^{\mathrm{abs}}+B_{\lambda_{f-1}}\left(T_{L}^{i+1}\right) Q_{\lambda_{f-1}}^{\mathrm{abs}}\right]\left(\lambda_{f}-\lambda_{f-1}\right)= \\
& \quad \frac{1}{4}\left(\frac{R_{*}}{r_{* L}}\right)^{2} \sum_{f=2}^{N_{\lambda}}\left[W_{* L}^{i, \lambda_{f}}+W_{* L}^{i, \lambda_{f-1}}\right]\left(\lambda_{f}-\lambda_{f-1}\right) \\
& \quad+\frac{1}{4} \sum_{K=1}^{N c} V_{K} n\left(\boldsymbol{x}_{K}\right) \frac{a^{2}}{r_{K L}^{2}} \sum_{f=2}^{N_{\lambda}}\left[W_{K L}^{i, \lambda_{f}}+W_{K L}^{i, \lambda_{f-1}}\right]\left(\lambda_{f}-\lambda_{f-1}\right) .
\end{aligned}
$$

\section{References}

Barber, P. W., \& Hill, S. C. 1990, Light Scattering by Particles: Computational Methods (World Scientific Publ. Co Pte Ltd)

Bouvier, J., Chelli, A., Allain, S., et al. 1999, A\&A, 349, 619

Bryden, G., Chen, X., Lin, D. N. C., Nelson, R. P., \& Papaloizou, C. B. 1999, ApJ, 514, 344

D’Angelo, G., Henning, Th., \& Kley, W. 2002, A\&A, 2002, 385, 647

Draine, B. T., \& Lee, H. M. 1984, ApJ, 285, 89

Draine, B. T. 1985, ApJS, 57, 587

Efstathiou, A., \& Rowan-Robinson, M. 1990, MNRAS, 245, 275

Egan, M. P., \& Shipman, R. F. 1995, AAS, 187, 100.05

Evans, A. 1994, The Dusty Universe (Wiley, New York)

Folini, D., \& Walder, E. 1999, IAUS, 193, 352

Folini, D., Walder, E., Psarros, M., \& Desboeufs, A. 2003, Stellar atmosphere modeling, PASP Conf. Ser., in press

Gordon, K. D., Misselt, K. A., Witt, A. N., \& Clayton, G. C. 2001, ApJ, 551, 269

Günther, R., \& Kley, W. 2002, A\&A, 387, 550

Ivezic, Z., \& Elitzur, M. 1995, ApJ, 445, 415

Ivezic, Z., Groenewegen, M. A. T., Men'shchikov, A., \& Szczerba, R. 1997, MNRAS, 291, 121

Misselt, K. A., Gordon, K. D., Clayton, G. C., \& Wolff, M. J. 2001, ApJ, 551, 277

Mie, G. 1908, Ann. Phys., 25

Ng, K. 1974, J. Chem. Phys., 61, 2680

Pascucci, I., Wolf, S., Steinacker, J., et al. 2003, A\&A, submitted

Pfalzner, S., Henning, Th., \& Kley, W. 2000, IAUS, 200, 193

Preibisch, Th., Ossenkopf, V., Yorke, H. W., \& Henning, Th. 1993, A\&A, 279, 577

Press, W. H., Teukolsky, S. A., Vetterling, W. T., \& Flannery, B. P. 1992, Numerical Recipes (Cambridge University Press, Cambridge)

Rouleau, F., \& Martin, P. G. 1991, ApJ, 377, 526

Steinacker, J., Thamm, E., \& Maier, U. 1996, JQSRT, 56, 1, 97

Steinacker, J., Hackert, R., Steinacker, A., \& Bacmann, A. 2002a, JQSRT, 74, 183

Steinacker, J., Bacmann, A., \& Henning, Th. 2002b, JQSRT, 75, 765

Stenholm, L. G., Störzer, H., \& Wehrse, R. 1991, JQSRT, 45, 1, 47

Uitenbroek, H. 2001, ApJ, 557, 389

Wolf, S., Henning, Th., \& Stecklum, B. 1999, A\&A, 349, 839

Wolf, S., \& Henning, Th. 1999, A\&A, 341, 675

Yorke, H. W. 1986, in Astrophysical Radiation Hydrodynamics, ed. K.-H. Winkler, \& M. L. Norman (Reidel, Dordrecht), 141 